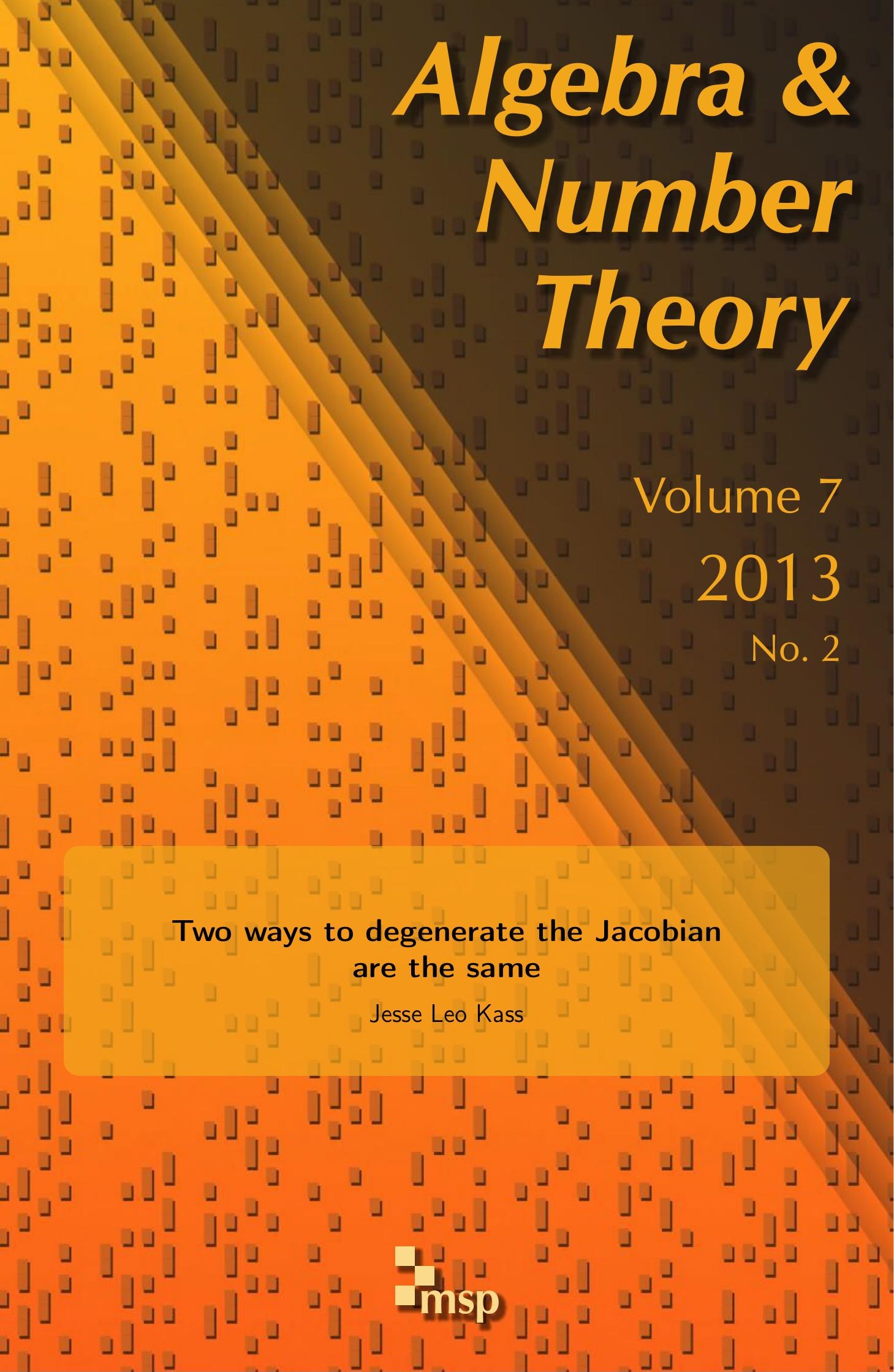




\title{
Two ways to degenerate the Jacobian are the same
}

\author{
Jesse Leo Kass
}

\begin{abstract}
We provide sufficient conditions for the line bundle locus in a family of compact moduli spaces of pure sheaves to be isomorphic to the Néron model. The result applies to moduli spaces constructed by Eduardo Esteves and Carlos Simpson, extending results of Busonero, Caporaso, Melo, Oda, Seshadri, and Viviani.
\end{abstract}

\section{Introduction}

1.1. Background. This paper relates two different approaches to extending families of Jacobian varieties. Recall that if $X_{0}$ is a smooth projective curve of genus $g$, then the associated Jacobian variety is a $g$-dimensional smooth projective variety $J_{0}$ that can be described in two different ways: as the universal abelian variety that contains $X_{0}$ (the Albanese variety), and as the moduli space of degree 0 line bundles on $X_{0}$ (the Picard variety). If $X_{U} \rightarrow U$ is a family of smooth, projective curves, then the Jacobians of the fibers fit together to form a family $J_{U} \rightarrow U$. In this paper, $U$ will be an open subset of a smooth curve $B$ (or, more generally, a Dedekind scheme), and we will be interested in extending $J_{U}$ to a family over $B$. Corresponding to the two different ways of describing the Jacobian (Albanese vs. Picard) are two different approaches to extending the family $J_{U} \rightarrow U$.

Viewing the Jacobian as the Albanese variety, it is natural to try to extend $J_{U} \rightarrow U$ by extending it to a family of group varieties over $B$. Néron [1964] showed that this can be done in a canonical way. He worked with an arbitrary family of abelian varieties $A_{U} \rightarrow U$ and proved that there is a unique $B$-smooth group scheme $\mathrm{N}:=\mathrm{N}\left(A_{U}\right) \rightarrow B$ extending $A_{U} \rightarrow U$ which is universal with respect to a natural mapping property. This scheme is called the Néron model. Arithmetic geometry has seen the use of the Néron model in a number of important results, e.g., [Mazur 1972; 1977; Mazur and Wiles 1984; Gross 1990]. The Néron model of a Jacobian variety plays a particularly prominent role, and an alternative description

The author was partially supported by NSF grant DMS-0502170.

MSC2010: primary 14H40; secondary 14L15, 14D22, 14H10.

Keywords: Néron model, stable sheaf, compactified Jacobian, relative Picard functor. 
of this scheme in terms of the relative Picard functor was given by Raynaud [1970]. We primarily work with Raynaud's description, which is recalled in Section 2.

An alternative approach, suggested by viewing the Jacobian as the Picard variety, is to extend $J_{U} \rightarrow U$ as a family of moduli spaces of sheaves. This approach was first proposed by Mayer and Mumford [1964]. Typically, one first extends $X_{U} \rightarrow U$ to a family of curves $X \rightarrow B$ and then extends $J_{U}$ to a family $\bar{J} \rightarrow B$ with the property that the fiber over a point $b \in B$ is a moduli space of sheaves on $X_{b}$ parametrizing certain line bundles, together with their degenerations. In this paper, we show that the line bundle locus $J$ in $\bar{J}$ is canonically isomorphic to the Néron model for some schemes $\bar{J}$ constructed in the literature.

To state this more precisely, we need to specify which schemes $\bar{J}$ we consider. The problem of constructing such a family of moduli spaces has been studied by many mathematicians, and they have constructed a number of different compactifications; see for example [Ishida 1978; D'Souza 1979; Oda and Seshadri 1979; Altman and Kleiman 1980; Caporaso 1994; Simpson 1994; Pandharipande 1996; Jarvis 2000; Esteves 2001]. Many of the difficulties to performing such a construction arise from the fact that, when $X_{b}$ is reducible, the degree 0 line bundles on a fiber $X_{b}$ do not form a bounded family.

For simplicity, assume the residue field $k(b)$ is algebraically closed and $X_{b}$ is reduced with components labeled $X_{1}, \ldots, X_{n}$. Given a line bundle $\mathcal{M}$ of degree 0 on $X_{b}$, the sequence $\left(\operatorname{deg}\left(\left.M\right|_{X_{1}}\right), \ldots, \operatorname{deg}\left(\left.M\right|_{X_{n}}\right)\right)$ is called the multidegree of $\mathcal{M}$. This sequence must sum to 0 , but may otherwise be arbitrary, which implies unboundedness. A bounded family can be obtained by fixing the multidegree, and typically the scheme $\bar{J}$ is defined so that it parametrizes (possibly coarsely) line bundles (and their degenerations) that satisfy a numerical condition on the multidegree. This paper focuses on constructions given by Simpson [1994] and by Esteves [2001], which we now describe in more detail.

For the Simpson moduli space, the numerical condition imposed on line bundles is slope semistabilty with respect to an auxiliary ample line bundle. This condition arises from the method of construction: the moduli space is constructed using geometric invariant theory (GIT), and slope stability corresponds to GIT stability. In general, the Simpson moduli space is a coarse moduli space in the sense that nonisomorphic sheaves may correspond to the same point of the space, but it contains an open subscheme (the stable locus) that is a fine moduli space, and we will work exclusively with this locus. Families of moduli spaces of semistable sheaves have been constructed for arbitrary families of projective schemes, but we will only be concerned with the case of families of curves.

The moduli spaces of Esteves parametrize sheaves that are $\sigma$-quasistable. Like slope stability, $\sigma$-quasistabillity is a numerical conditions on the multidegree, but it is defined in terms of an auxiliary vector bundle $\mathscr{E}$ and a section $\sigma$, rather than an 
ample line bundle. The moduli spaces are constructed for families over an arbitrary locally noetherian base, but strong conditions are required of the fibers: They must be geometrically reduced. The space is constructed as a closed subspace of a (nonnoetherian, nonseparated) algebraic space that was constructed in [Altman and Kleiman 1980]. For nodal curves, Melo and Viviani [2012] describe the relation between the Esteves moduli spaces and the Simpson moduli spaces. However, here we treat these moduli spaces separately.

For a discussion of the relation between these moduli spaces and other moduli spaces constructed in the literature, the reader is directed to [Alexeev 2004; Casalaina-Martin et al. 2011, Section 2]. The reader familiar with the work of Caporaso is warned of one potential point of confusion. In [Caporaso 1994], the compactified Jacobian associated to a stable curve $X$ parametrizes pairs $(Y, L)$ consisting of a line bundle $L$ on a nodal curve $Y$ stably equivalent to $X$ that satisfies certain conditions. The line bundle locus $J$ that we study corresponds to the locus parametrizing pairs $(Y, L)$ with $X=Y$.

1.2. Main result. The main result of this paper relates the line bundle locus in a proper family of moduli spaces of sheaves to the Néron model of the Jacobian:

Theorem 1. Fix a Dedekind scheme B. Let $f: X \rightarrow B$ be a family of geometrically reduced curves with regular total space $X$ and smooth generic fiber $X_{\eta}$. Let $J \subset \bar{J}$ the locus of line bundles in one of the following moduli spaces:

- the Esteves compactified Jacobian $\bar{J}_{\mathscr{E}}^{\sigma}$;

- the Simpson compactified Jacobian $\bar{J}_{\mathscr{L}}^{0}$ associated to an $f$-ample line bundle $\mathscr{L}$ such that slope semistability coincides with slope stability.

Then $J$ is the Néron model of its generic fiber.

Theorem 1 is the combination of Corollaries 4.2 and 4.5, which themselves are consequences of Theorem 3.9. Theorem 3.9 is quite general, and we expect that it applies to many other fine moduli spaces of sheaves (but not coarse ones). In particular, Theorem 3.9 applies to families of curves with possibly nonreduced fibers, though then general results asserting the existence of a suitable moduli space are unknown (but see Section 4.3 for some simple examples).

The arithmetically inclined reader should note Theorem 1 and the results later in this paper do not place any hypotheses on the base Dedekind scheme $B$. In particular, we do not assume that the residue fields are perfect. This surprised the author initially as there is a body of work (e.g., [Liu et al. 2004; Raynaud 1970]) showing that various pathologies can arise when $k(b)$ fails to be perfect.

Theorem 1 has interesting consequences for both the Néron model and the compactified Jacobian. One consequence of the theorem is that Néron models of Jacobians can often be constructed over high-dimensional bases. The Néron 
model of an abelian variety is only defined over a (regular) 1-dimensional base $B$, but no such dimensional hypotheses are needed to apply the existence results from [Simpson 1994; Esteves 2001]. At the end of Section 4.3, we examine a family $J \rightarrow \mathbb{P}^{2}$ over the plane with the property that a dense, open subset of $\mathbb{P}^{2}$ is covered by lines $C$ such that the restriction $J_{C}$ of $J$ is the Néron model of its generic fiber. Surprisingly, while the Néron models fit into a 2-dimensional family, their group structure does not.

Theorem 1 also has interesting consequences for the moduli spaces of Esteves and Simpson. Indeed, if $f: X \rightarrow B$ is a family of curves satisfying the hypotheses of the theorem, then both the Esteves Jacobians $J_{\mathscr{E}}^{\sigma}$ and the Simpson Jacobians $J_{\mathscr{L}}^{0}$ (for $\mathscr{L}$ as in the hypothesis) are independent of the particular polarizations, and every such Simpson Jacobian is isomorphic to every Esteves Jacobian. This is not immediate from the definitions. At the end of Section 4.1, we discuss this fact in greater detail and pose a related question.

1.3. Past results. Certain cases of Theorem 1 were already known. In his (unpublished) thesis, Simone Busonero [2008] established Theorem 1 for certain Esteves Jacobians. A different proof using similar techniques that extends the result to the Simpson moduli spaces is due to Melo and Viviani [2012, Theorem 3.1]. They prove Theorem 1 when the fibers of $f$ are nodal and $X$ is regular. We do not discuss the Caporaso universal compactified Jacobian here, but the relation between that scheme and the Néron model was described by Caporaso [2008a; 2008b; 2012, especially Theorem 2.9]. Earlier still, Oda and Seshadri related their $\phi$-semistable compactified Jacobians, also not discussed here, to Néron models [Oda and Seshadri 1979, Corollary 14.4]. In each of those papers, an important step in the proof is a combinatorial argument establishing that, for example, the natural map from the set of $\sigma$-quasistable multidegrees to the degree class group is a bijection.

The proof given here does not use any combinatorics, and the idea can be described succinctly. Consider the special case where $B:=\operatorname{Spec}(\mathbb{C} \llbracket t \rrbracket)$, which is a strict henselian discrete valuation ring with algebraically closed residue field. There is a natural map $J \rightarrow \mathrm{N}$ to the Néron model coming from the universal property of $\mathrm{N}$, and an application of Zariski's main theorem shows that this morphism is an open immersion. Thus the only issue is set-theoretic surjectivity. Because $B$ is henselian, every point on the special fiber of $\mathrm{N}$ is the specialization of a section, so surjectivity is equivalent to the surjectivity of the map $J(\mathbb{C} \llbracket t \rrbracket) \rightarrow J(\operatorname{Frac} \mathbb{C} \llbracket t \rrbracket)$ that sends a section to its restriction to the generic fiber. A given point $p \in J($ Frac $\mathbb{C} \llbracket t \rrbracket)$ may be extended to a section $\sigma \in \bar{J}(\mathbb{C} \llbracket t \rrbracket)$ of $\bar{J}$ by the valuative criteria. As $\bar{J}$ is a fine moduli space, $\sigma$ corresponds to a family of rank 1, torsion-free sheaves, which in fact must be a family of line bundles because $X$ is factorial. We may conclude that $\sigma \in J(\mathbb{C}[t])$, yielding the result. 
1.4. Questions. It would be interesting to know when a Simpson Jacobian $J_{\mathscr{L}}^{0}$ satisfying the hypotheses of Theorem 1 exists; that is, given a family $f: X \rightarrow B$, does there exist an ample line bundle $\mathscr{L}$ such that every $\mathscr{L}$-slope semistable sheaf of degree 0 is stable? We briefly survey the literature on this question at the end of Section 4.2.

More generally, given a family $f: X \rightarrow B$, it would be desirable to have a description of the maximal subfunctors of the degree 0 relative Picard functor $\mathrm{P}^{0}$ representable by a separated $B$-scheme. We discuss this question in Section 4.3, where we analyze the simple case of genus 1 curves.

1.5. Organization. We end this introduction with a few technical remarks about the paper. The moduli spaces of sheaves that we consider are moduli spaces of pure sheaves. On a curve, a coherent sheaf is pure if and only if it is Cohen-Macaulay. This condition is also equivalent to the condition of being torsion-free in the sense of elementary algebra when the curve is integral, and the term "torsion-free" is sometimes used in place of "pure".

The term "family of curves" only to refers to families with geometrically irreducible generic fibers. This is done to avoid notational complications concerning multidegrees. Families of curves are required to be proper, but not projective. A family of curves over a Dedekind scheme can fail to be projective (e.g., [Altman and Kleiman 1980, 8.10]), but projectivity is automatic if the local rings of the total space are factorial, which is the main case of interest. (See Proposition 4.1.)

We prove the main results for families over a base scheme $S$ that is the spectrum of a strict henselian discrete valuation ring rather than a more general Dedekind scheme. Doing so lets us make sectionwise arguments because a smooth family of a henselian base admits many sections. Furthermore, this is not a real restriction: Results over a general Dedekind base can be deduced by passing to the strict henselization.

The body of the paper is organized as follows. In Section 2, we review Rayanud's construction of the maximal separated quotient. We then relate this scheme to a general moduli space of line bundles satisfying some axioms in Section 3. Finally, we describe some schemes that satisfy these axioms in the final section, Section 4.

\section{Conventions}

1.1. The symbol $X_{T}$ denotes the fiber product $X \times_{S} T$.

1.2. The letter $S$ denotes the spectrum of a strict henselian discrete valuation ring with special point 0 and generic point $\eta$.

1.3. A curve over a field $k$ is a proper $k$-scheme $f_{0}: X_{0} \rightarrow \operatorname{Spec}(k)$ that is geometrically connected and of pure dimension 1 . 
1.4. If $B$ is a scheme, then a family of curves over $B$ is a proper, flat morphism $f$ : $X \rightarrow B$ whose fibers are curves and whose geometric generic fibers are irreducible.

1.5. If $f: Y \rightarrow B$ is a finitely presented morphism, then we write $Y^{\text {sm }} \subset Y$ for the smooth locus of $f$.

1.6. A coherent module $I_{0}$ on a noetherian scheme $X_{0}$ is rank 1 if the localization of $I_{0}$ at $x$ is isomorphic to $\mathbb{O}_{X_{0}, x}$ for every generic point $x$.

1.7. A coherent module $I_{0}$ on a noetherian scheme $X_{0}$ is pure if the dimension of $\operatorname{Supp}\left(I_{0}\right)$ equals the dimension of $\operatorname{Supp}\left(J_{0}\right)$ for every nonzero subsheaf $J_{0}$ of $I_{0}$.

1.8. If $X_{0} \rightarrow \operatorname{Spec}(k)$ is proper, then the degree of a coherent $O_{X_{0}}$-module $\mathscr{F}$ is defined by $\operatorname{deg}(\mathscr{F}):=\chi(\mathscr{F})-\chi\left(\mathscr{O}_{X}\right)$.

\section{Raynaud's maximal separated quotient}

We begin by reviewing Raynaud's construction of the Néron model of a Jacobian and, more generally, the maximal separated quotient of the relative Picard functor [Raynaud 1970]. Much of this material is also treated in [Bosch et al. 1990, Chapter 9].

Let $S$ be a strict henselian discrete valuation ring with generic point $\eta$ and special point 0 . Given a family of curves $f: X \rightarrow S$, the relative Picard functor $\mathrm{P}$ of $f$ is defined to be the étale sheaf $\mathrm{P}: S$-Sch $\rightarrow$ Grp associated to the functor

$$
T \mapsto \operatorname{Pic}\left(X_{T}\right) .
$$

Here $\operatorname{Pic}\left(X_{T}\right)$ is the set of isomorphism classes of line bundles on $X_{T}$. Rayanud actually defines $P$ to be the associated fppf sheaf, but then observes that this is the same as the associated étale sheaf ([Raynaud 1970, 1.2]; see also [Kleiman 2005, Remark 9.2.11]). The fibers of $\mathrm{P}$ are representable by group schemes locally of finite type, and $\mathrm{P}$ itself is representable by an algebraic space if and only if $f$ is cohomologically flat [Raynaud 1970, Theorem 5.2]. Regardless of its representability properties, $\mathrm{P}$ is locally finitely presented and formally $S$-smooth.

Inside of P, we may consider the functor E : $S$-Sch $\rightarrow$ Grp that is defined to be the scheme-theoretic closure of the identity section. This is the fppf subsheaf of P generated by the elements $g \in \mathrm{P}(T)$, where $T \rightarrow S$ is flat and $g_{\eta} \in \mathrm{P}\left(T_{\eta}\right)$ is the identity element. When $\mathrm{P}$ is a scheme, this coincides with the usual notion of closure. The representability properties of $\mathrm{E}$ are similar to those of $\mathrm{P}$ : The fibers of $\mathrm{E}$ are group schemes locally of finite type, and $\mathrm{E}$ is representable by an algebraic space precisely when $f$ is cohomologically flat [Raynaud 1970, Proposition 5.2]. When representable, E $\rightarrow S$ is an étale $S$-group space; in general, the generic fiber of $\mathrm{E}$ is the trivial group scheme, and the special fiber is a group scheme of dimension equal to $h^{0}\left(\mathrm{O}_{X_{0}}\right)-h^{0}\left(\mathrm{O}_{X_{\eta}}\right)$. 
When E is not the trivial $S$-group scheme, P does not satisfy the valuative criteria of separatedness. We can, however, form the maximal separated quotient $\mathrm{Q}: S$-Sch $\rightarrow$ Grp of P. By definition, this is the fppf quotient sheaf Q := P / E. The maximal separated quotient $\mathrm{Q}$ is always representable by a scheme that is $S$-smooth, separated, and locally of finite type [Raynaud 1970, Theorem 4.1.1, Proposition 8.0.1]. Rather than working directly with Q, we shall primarily work with the slightly smaller subfunctor $\mathrm{Q}^{\tau}: S$-Sch $\rightarrow$ Grp, which we now define.

Suppose generally that $B$ is a scheme and $G: S$-Sch $\rightarrow$ Grp is a $B$-group functor whose fibers are representable by group schemes locally of finite type. For every point $b \in B$, we may form the identity component $G_{b}^{\mathrm{o}} \subset G_{b}$ and the component

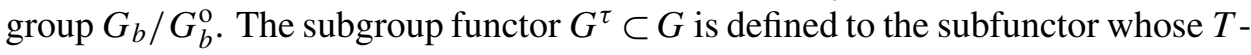
valued points are elements $g \in G(T)$ with the property that, for every $t \in T$ mapping to $b \in B$, the element $g_{t} \in G_{b}(k(t))$ maps to a torsion element of $G_{b} / G_{b}^{\mathrm{o}}(k(t))$. If we instead require that $g_{t}$ maps to the identity element, then we obtain the subgroup functor $G^{\mathrm{o}} \subset G$. Let us examine these constructions when $B$ equals $S$ and $G$ equals $\mathrm{P}$ or $\mathrm{Q}$.

The functors $\mathrm{P}^{\mathrm{o}}$ and $\mathrm{P}^{\tau}$ coincide, and this common functor is the étale sheaf associated to the assignment sending $T$ to the set of isomorphism classes of line bundles on $X_{T}$ that fiberwise have multidegree 0. From this description, it is easy to see that $\mathrm{P}^{\mathrm{o}}=\mathrm{P}^{\tau} \subset \mathrm{P}$ is an open subfunctor. Another open subfunctor of $\mathrm{P}$ is the subfunctor parametrizing line bundles on $X_{T}$ with fiberwise degree 0 , which we denote by $\mathrm{P}^{0}$. It is typographically difficult to distinguish between $\mathrm{P}^{0}$ and $\mathrm{P}^{\mathrm{o}}$, but we will not make use of $\mathrm{P}^{\mathrm{O}}$ in this paper, so this should not cause confusion.

The functors $\mathrm{Q}^{\circ}$ and $\mathrm{Q}^{\tau}$ are different in general. They are, however, both open subfunctors of Q [Grothendieck 1966b, Theorem 1.1(i.i), Corollary 1.7]. In particular, they are both representable by smooth and separated $S$-group schemes that are locally of finite type. In fact, both schemes are of finite type over $S$ as their fibers are easily seen to have a finite number of connected components. The condition that $\mathrm{Q}^{\tau} \subset \mathrm{Q}$ is a closed subscheme is important, but slightly subtle. A characterization of this condition is given by [Raynaud 1970, Proposition 8.1.2(iii)]; one sufficient (but not necessary) condition for $\mathrm{Q}^{\tau} \subset \mathrm{Q}$ to be closed is that the local rings of $X$ are factorial.

The factoriality condition is also almost sufficient to ensure that $\mathrm{Q}^{\tau}$ is the Néron model of its generic fiber. Suppose that the generic fiber of $f$ is smooth, so the generic fiber of $\mathrm{Q}^{\tau} \rightarrow S$ is an abelian variety, and thus it makes sense to speak of the Néron model $\mathrm{N}:=\mathrm{N}\left(\mathrm{Q}_{\eta}^{\tau}\right)$. By the universal property, there is a unique morphism $\mathrm{Q}^{\tau} \rightarrow \mathrm{N}$ that is the identity on the generic fiber. Theorem 8.1.4 of [Raynaud 1970] states that if the local rings of $X$ are factorial, then $\mathrm{Q}^{\tau} \rightarrow \mathrm{N}$ is an isomorphism in the cases that $k(0)$ is perfect and that a certain invariant $\delta$ is coprime to the residual characteristic. 
The proof uses the characterization of the Neron model in terms of the weak Néron mapping property. Recall that a $S$-scheme $Y \rightarrow S$ is said to be a weak Néron model of its generic fiber if the natural map $Y(S) \rightarrow Y(\eta)$ is bijective. If $G \rightarrow S$ is a finite type $S$-group scheme whose generic fiber is an abelian variety, then $G$ is the Néron model of its generic fiber if and only if it satisfies the weak Néron mapping property [Bosch et al. 1990, Section 7.1, Theorem 1].

\section{The main theorem}

Here we derive the main results for families over a strict henselian discrete valuation ring $S$ with generic point $\eta$ and special point 0 . Specifically, we provide sufficient conditions for the maximal separated quotient $\mathrm{Q}^{\tau}$ of the Picard functor to be the Néron model and we relate $\mathrm{Q}^{\tau}$ to a fine moduli space of line bundles that satisfies certain axioms. These moduli spaces are, by definition, subfunctors of a (large) functor that we now define.

Definition 3.1. If $T$ is a $S$-scheme, then we define $\operatorname{Sheaf}\left(X_{T}\right)$ to be the set of isomorphism classes of $O_{T}$-flat, finitely presented $O_{X_{T}}$-modules $\mathscr{I}$ on $X_{T}$ that are fiberwise pure, rank 1, and of degree 0 .

The functor $\mathrm{Sh}=\mathrm{Sh}_{X / S}: S$-Sch $\rightarrow$ Sets is defined to be the étale sheaf associated to the functor

$$
T \mapsto \operatorname{Sheaf}\left(X_{T}\right) .
$$

There is a tautological transformation $\mathrm{P}^{0} \rightarrow$ Sh that realizes $\mathrm{P}^{0}$ as a subfunctor of Sh.

Lemma 3.2. The subfunctor $\mathrm{P}^{0} \subset \mathrm{Sh}$ is open.

Proof. Given a $S$-scheme $T$ and a morphism $g: T \rightarrow$ Sh, we must show that $T \times{ }_{\mathrm{Sh}} \mathrm{P}^{0}$ is representable by a scheme and that $T \times{ }_{\mathrm{Sh}} \mathrm{P}^{0} \rightarrow T$ is an open immersion. Thus, let $g$ be given.

By definition, there exists an étale surjection $p: T^{\prime} \rightarrow T$ and a sheaf $\Phi^{\prime} \in$ Sheaf $\left(X_{T^{\prime}}\right)$ that represents $g \circ p: T^{\prime} \rightarrow$ Sh. Consider the subset $U^{\prime} \subset T^{\prime}$ of points $t \in T^{\prime}$ with the property that the restriction of $\mathscr{I}$ to the fiber $X_{t}$ is a line bundle. This locus is open by [Altman and Kleiman 1980, Lemma 5.12(a)], and one may easily show that $U^{\prime}$ represents $T^{\prime} \times{ }_{\mathrm{Sh}} \mathrm{P}^{0}$. A descent argument establishes the analogous property for the image $U$ of $U^{\prime}$ under $T^{\prime} \rightarrow T$. This completes the proof.

A remark about topologies: We work with the étale sheaf associated to (3-1), but one could instead work with the associated fppf sheaf. When $f$ is projective, it is a theorem of Altman and Kleiman [1980, Theorem 7.4] that the subfunctor of Sh parametrizing simple sheaves can be represented by a quasiseparated, locally finitely presented $S$-algebraic space, and hence is an fppf sheaf. We do not know if Sh is an fppf sheaf in general. Here Sh is just used as a tool for keeping track of 
representable functors, and certainly any representable subfunctor of Sh is an fppf sheaf.

One reason for working with the étale topology instead of the fppf topology is that it makes the following fact easy to prove.

Fact 3.3. The natural map Sheaf $(X) \rightarrow \operatorname{Sh}(S)$ is surjective.

Proof. Let $g \in \operatorname{Sh}(S)$ be given. By definition, there is an étale morphism $S^{\prime} \rightarrow S$ and an element ${I^{\prime}}^{\prime} \in \operatorname{Sheaf}\left(X_{S^{\prime}}\right)$ that maps to $g_{S^{\prime}} \in \operatorname{Sh}\left(S^{\prime}\right)$. But $S$ is strict henselian, so $S^{\prime} \rightarrow S$ may be taken to be an isomorphism $S \rightarrow S$ [Grothendieck 1967, Proposition 18.8.1(c)], in which case the result is obvious.

The following two facts about separably closed fields are standard, but they will be used so frequently that it is convenient to record them.

Fact 3.4. If $k(0)$ is a separably closed field and $f_{0}: Y_{0} \rightarrow \operatorname{Spec}(k(0))$ is smooth of relative dimension $n$, then the closed points of $Y_{0}$ with residue field $k(0)$ are dense.

Proof. This is [Bosch et al. 1990, Corollary 13]. The scheme $Y_{0}$ can be covered by affine opens $U_{0}$ that admit an étale morphism $p: U_{0} \rightarrow \mathbb{A}_{k(0)}^{n}$. Certainly, the closed points with residue field $k(0)$ are dense in the image of $p$. If $v_{0} \in \mathbb{A}_{k(0)}^{n}$ is one such point, then $p^{-1}\left(v_{0}\right)$ is a finite, étale $k(0)$-algebra, and hence a disjoint union of closed points defined over $k(0)$. Density follows.

Fact 3.4 is typically used in conjunction with the following fact to assert that a smooth morphism has many sections.

Fact 3.5. Let $Y \rightarrow S$ be a smooth morphism over strict henselian discrete valuation ring. Then $Y(S) \rightarrow Y(k(0))$ is surjective.

Proof. This is [Grothendieck 1967, Corollary 17.17.3], or [Bosch et al. 1990, Proposition 14]. If $U$ and $X^{\prime}$ are as in the statement of the former, then we must have $U=S$ and $X^{\prime} \rightarrow U$ may be taken to be an isomorphism (again, by [Grothendieck 1967, Proposition 18.8.1(c)]).

We now prove the main results of the paper.

Proposition 3.6. Let $f: X \rightarrow S$ be a family of curves and $J \subset \mathrm{P}^{0}$ a subfunctor such that the generic fibers $J_{\eta}=\mathrm{P}_{\eta}^{0}$ coincide. Assume $J$ is represented by a smooth, finitely presented $S$-scheme.

If $J$ is $S$-separated, then $J \rightarrow \mathrm{Q}$ is an open immersion. Furthermore, the image is contained in $\mathrm{Q}^{\tau}$ provided $\mathrm{Q}^{\tau} \subset \mathrm{Q}$ is closed (e.g., the local rings of $X$ are factorial). 
Proof. This is an application of Zariski's main theorem. We begin by showing that the induced map $J \rightarrow \mathrm{Q}$ is injective on closed points. It is enough to verify this after extending base $S$ so that $k(0)$ is algebraically closed. Thus, we will temporarily assume $k:=k(0)$ is algebraically closed and work with $k$-valued points instead of closed points. Given $q \in \mathrm{Q}(k)$, there is nothing to show when the fiber over $q$ is empty. If nonempty, pick $p \in J(k)$ mapping to $q$. We may invoke Fact 3.5 to assert that there exists a section $\sigma \in J(S)$ with $\sigma(0)=p$.

The fiber of $\mathrm{P} \rightarrow \mathrm{Q}$ over $q$ is the set of elements of the form $p+e$ with $e \in \mathrm{E}(k)$ or, equivalently, the elements of $(\sigma+\mathrm{E})(k)$ [Raynaud 1970, Corollary 4.1.2]. Restricting to $J$, we see that the fiber of $q$ under $J \rightarrow \mathrm{Q}$ is the set of $k$-valued points of $(\sigma+\mathrm{E}) \cap J$. But $(\sigma+\mathrm{E}) \cap J$ is the scheme-theoretic closure of $\sigma$ in $J$ (by [Grothendieck 1965, 2.8.5]), which is just the image of $\sigma$ by separatedness. In particular, the preimage of $q$ under $J \rightarrow \mathrm{Q}$ must be the singleton set $\{p\}$. This proves that the map is injective on closed points. We now return to the case where $S$ is a henselian discrete valuation ring (so $k(0)$ is no longer assumed to be algebraically closed).

It follows that the set-theoretic fibers of $J \rightarrow \mathrm{Q}$ are finite sets. Indeed, if $Z \subset J$ is the locus of points $x \in J$ with the property that $x$ lies in a positive dimensional fiber, then $Z$ is closed by Chevalley's theorem [Grothendieck 1965, 13.1.3]. Furthermore, $Z$ is contained in the special fiber $J_{0}$ and contains no closed points. This is only possible if $Z$ is the empty scheme. In other words, the set-theoretic fibers of $J \rightarrow$ Q are 0-dimensional, and hence finite (by [Grothendieck 1964, 14.1.9]).

It follows immediately from Zariski's main theorem [Grothendieck 1961, 4.4.9] that $J \rightarrow$ Q is an open immersion. This proves the first part of the theorem. To complete the proof, observe that flatness implies that the generic fiber of $J_{\eta}$ is dense in $J$ [Grothendieck 1965, 2.8.5]. In particular, $J$ is contained in the closure of $J_{\eta}$ in $\mathrm{Q}$. The generic fiber of $J$ coincides with the generic fiber of $\mathrm{Q}^{\tau}$, so the closure of this common scheme is contained in $\mathrm{Q}^{\tau}$ when $\mathrm{Q}^{\tau} \subset \mathrm{Q}$ is closed. This completes the proof.

Remark 3.7. In Proposition 3.6, we do not assume that $J \subset \mathrm{P}^{0}$ is an open subfunctor, but this condition holds in most cases of interest. When open, $J$ is automatically formally smooth and locally of finite presentation. Thus, the key hypothesis in the proposition is that $J$ is represented by a $S$-separated scheme. A similar remark holds for Theorem 3.9; there the key hypotheses are that $\bar{J}$ satisfies the valuative criteria of properness and that $J$ is representable. Indeed, we do not even need to assume that $\bar{J}$ is representable.

Under stronger assumptions, we can actually show that the natural map $J \rightarrow \mathrm{Q}^{\tau}$ is an isomorphism. The essential point is to prove that $J$ satisfies the weak Néron mapping property. When $J$ can be embedded in a $S$-proper moduli space $\bar{J}$, this 
property holds provided that the local rings of $X$ are factorial. The content of this claim is that a line bundle on the generic fiber can specialize only to a line bundle on the special fiber. By localizing, the claim is equivalent to the following lemma, which is based on a proof from [Altman and Kleiman 1979, p. 27 after Step XII].

Lemma 3.8. Suppose that $(R, \pi)$ is a discrete valuation ring and $R \rightarrow 0$ a local, flat algebra extension with 0 noetherian. Let $M$ be a $R$-flat, finite 0 -module with the property that $M\left[\pi^{-1}\right]$ is free of rank 1 and $M / \pi M$ is a rank 1 , pure module. If 0 is factorial, then $M$ is free of rank 1.

Proof. We can certainly assume 0 is not the zero ring. To ease notation, we write $\bar{M}:=M / \pi M$ and $\overline{0}:=\mathbb{O} / \pi \mathcal{O}$. It is enough to prove that $M$ is isomorphic to a height 1 ideal. Indeed, such an ideal is principal by the factoriality assumption.

We argue by first showing that $M$ is isomorphic to an ideal of $\mathbb{O}$. Let $\overline{\mathfrak{p}}_{1}, \ldots, \overline{\mathfrak{p}}_{n}$ be the minimal primes of $\overline{\mathcal{O}}$ and $\mathfrak{p}_{1}, \ldots, \mathfrak{p}_{n}$ the corresponding primes of $\mathbb{O}$. We have assumed that the stalk $\bar{M} \otimes k\left(\overline{\mathfrak{p}}_{i}\right)$ is 1-dimensional. This stalk coincides with the stalk $M \otimes k\left(\mathfrak{p}_{i}\right)$, so we can conclude that the localization $M_{\mathfrak{p}_{i}}$ is free of rank 1 for $i=1, \ldots, n$.

We can also conclude that the same holds for the localizations of the dual module $M^{\vee}:=\operatorname{Hom}(M, \mathcal{O})$. An application of the prime avoidance lemma shows that there exists an element $\phi \in M^{\vee}$ that maps to a generator of $M_{\mathfrak{p}_{i}}^{\vee}$ for all $i$. We will show that $\phi: M \rightarrow \mathcal{O}$ realizes $M$ as a $R$-flat family of ideals (i.e., $\phi$ is injective with $R$-flat cokernel).

It is enough to show that the reduction $\bar{\phi}: \bar{M} \rightarrow \overline{\mathcal{O}}$ is injective. An element of the kernel of this map is also in the kernel of the composition

$$
\bar{M} \rightarrow \bigoplus \bar{M}_{\overline{\mathfrak{p}}_{i}} \rightarrow \bigoplus \overline{\mathcal{O}}_{\overline{\mathfrak{p}}_{i}} .
$$

The kernel of the leftmost map is a submodule whose support does not contain any of the primes $\overline{\mathfrak{p}}_{i}$, and thus must be zero by pureness. Furthermore, the rightmost map is an isomorphism by construction. This proves injectivity.

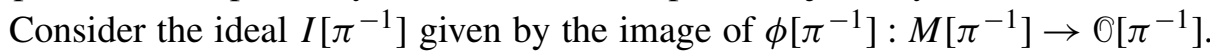
This is a principal ideal, and hence is either the unit ideal or an ideal of height at most 1 (Hauptidealsatz!). By flatness, the same is true of the image $I$ of $\phi$. In fact, $I$ cannot be a height zero ideal: The only such prime is the zero ideal, which does not satisfy the hypotheses. Thus, $I$ is either the unit ideal or a height 1 ideal. In either case, $I$ must be principal, and the proof is complete.

We record the factorial condition as a hypothesis.

Hypothesis 1. We say a family of curves $f: X \rightarrow B$ over a Dedekind scheme satisfies Hypothesis 1 if the generic fiber $X_{\eta}$ is smooth and the local rings of $X_{S}$ are factorial for every strict henselization $S \rightarrow B$. 
Hypothesis 1 is satisfied when $X$ is regular and $X_{\eta}$ is smooth. We now prove the main theorem of this paper.

Theorem 3.9. Let $f: X \rightarrow S$ be a family of curves and $\bar{J}$ a subfunctor of Sh such that the generic fibers $\bar{J}_{\eta}=\mathrm{Sh}_{\eta}$ coincide. Assume the line bundle locus $J \subset \bar{J}$ is represented by a smooth and finitely presented $S$-scheme.

If $\bar{J}$ satisfies the valuative criteria of properness and $f$ satisfies Hypothesis 1 , then $\mathrm{Q}^{\tau}$ is the Néron model and

$$
J \subset \mathrm{Q}^{\tau}=\mathrm{N}
$$

is an open subscheme that contains all the $k(0)$-valued points of $\mathrm{Q}^{\tau}$. Furthermore,

$$
J=\mathrm{Q}^{\tau}=\mathrm{N}
$$

provided one of the following conditions hold:

(1) $k(0)$ is algebraically closed;

(2) $J$ is stabilized by the identity component $\mathrm{Q}^{\mathrm{o}}$.

Proof. By Proposition 3.6, the natural map $J \rightarrow$ Q is an open immersion with image contained in $\mathrm{Q}^{\tau}$. Using this fact, we can prove that $\mathrm{Q}^{\tau}$ is the Néron model of its generic fiber. Indeed, it is enough to prove that $\mathrm{Q}^{\tau}$ satisfies the weak Néron mapping property. The open subscheme $J \subset \mathrm{Q}^{\tau}$, in fact, already satisfies this property. Let $\sigma_{\eta} \in \mathrm{Q}^{\tau}(\eta)=J(\eta)$ be given. By properness, we can extend $\sigma_{\eta}$ to a section $\sigma \in \bar{J}(S)$, and this element can be represented by a family $\Phi$ of pure, rank 1 sheaves (by Fact 3.3). But every such family is a family of line bundles (Lemma 3.8), and hence $\sigma$ lies in $J(S) \subset \bar{J}(S)$. In other words, $J$ satisfies the weak Néron mapping property.

The weak Néron mapping property of $J$ also implies that the image of $J$ contains all the $k(0)$-valued points of $\mathrm{Q}^{\tau}$. Indeed, every $k(0)$-valued point of $\mathrm{Q}^{\tau}$ is the specialization of a section by Fact 3.5. If $k(0)$ is algebraically closed, then we have shown that $J$ contains every $k(0)$-valued point of $\mathrm{Q}^{\tau}$, hence every closed point. Thus, $J=\mathrm{Q}^{\tau}$, and there is nothing more to show.

Let us now turn our attention to the case where $k(0)$ is only separably closed, but $J$ is stabilized by $\mathrm{Q}^{\circ}$. Our goal is to show $J=\mathrm{Q}^{\tau}$, and to show this, we pass to the special fiber $J_{0} \rightarrow \mathrm{Q}_{0}^{\tau}$ and argue with points. Let $x$ be a $\bar{k}(0)$-valued point of $\mathrm{Q}^{\tau}$, where $\bar{k}(0)$ is the algebraic closure of the residue field. By density (Fact 3.4), there exists a $\bar{k}(0)$-valued point $y$ in the image of $J_{0} \rightarrow \mathrm{Q}_{0}^{\tau}$ that lies in the same connected component as $x$. We have $x=y+(x-y)$, which expresses $x$ as the sum of a point of $\mathrm{Q}_{0}^{\mathrm{o}}$ and a point of $J_{0}$. The point $x$ must lie in $J_{0}$ by assumption. This shows that the image of $J$ contains all of $\mathrm{Q}^{\tau}$, completing the proof. 
Remark 3.10. The hypothesis that $J$ is stabilized by the identity component $\mathrm{Q}^{0}$ is perhaps unexpected, but it is often satisfied in practice. The moduli space $\bar{J}$ is typically constructed by imposing numerical conditions on the multidegree of a sheaf, and the multidegree is invariant under the action of $\mathrm{Q}^{\circ}$ (because the action is given by tensoring with a multidegree 0 line bundle).

In the next section, we will show that certain moduli spaces constructed in the literature satisfy the hypotheses of Theorem 3.9. There are, however, families of curves $f: X \rightarrow S$ with factorial local rings $\mathrm{O}_{X, x}$ such that there does not exist a $S$-scheme $\bar{J}$ satisfying the conditions of the theorem. Indeed, the family $f: X \rightarrow S$ in [Raynaud 1970, Example 9.2.3] is a family of genus 1 curves such that the local rings of $X$ are factorial (even regular), but the natural map $\mathrm{Q}^{\tau} \rightarrow \mathrm{N}$ is not an isomorphism. In particular, no $\bar{J}$ satisfying the hypotheses of Theorem 3.9 can exist.

\section{Applications}

Here we apply Theorem 3.9 to some families of moduli spaces from the literature and then deduce consequences. The two moduli spaces that we are interested in are the Esteves moduli space of quasistable sheaves (Section 4.1) and the Simpson moduli space of slope stable sheaves (Section 4.2). In Section 4.3, we discuss the special case of families of genus 1 curves, where suitable moduli spaces can be constructed explicitly.

The moduli spaces we study are associated to a relatively projective family of curves. We are primarily interested in families over a Dedekind scheme with locally factorial total space, in which case projectivity is automatic. This fact is a consequence of the generalized Chevalley Conjecture when the Dedekind scheme is defined over a field, but we do not know a reference. For completeness, we prove:

Proposition 4.1. Let $f: X \rightarrow B$ be a family of curves over a Dedekind scheme. If the local rings of $X$ are factorial, then $f$ is projective.

Proof. This proof was explained to the author by Steven Kleiman. Fix a closed point $b_{0} \in B$. Given any component $F \subset X_{b_{0}}$, I claim that we can find a line bundle $\mathscr{L}$ on $X$ that has nonnegative degree on every component of every fiber and strictly positive degree on $F$.

Pick a closed point $x \in F$ and an open affine neighborhood $U \subset X$ of that point. By the prime avoidance lemma, we can find a regular function $r \in H^{0}\left(U, \mathcal{O}_{X}\right)$ that does not vanish on any component of $X_{b_{0}}$ but does vanish at $x$. Pick a component $D$ of the closure of $\{r=0\} \subset U$ in $X$. Then $D$ is a Cartier divisor (by the factoriality assumption) that does not contain any component of any fiber $X_{b}$ (by construction). 
Furthermore, $D$ has nontrivial intersection with $F$. The associated line bundle $\mathscr{L}:=\mathrm{O}_{X}(D)$ has the desired positivity property.

Now construct one such line bundle for every irreducible component $F$ of $X_{b_{0}}$ and define $\mathcal{M}$ to be their tensor product. The line bundle $\mathcal{M}$ is nef on every fiber and ample on $X_{b_{0}}$. Ampleness is an open condition, so $\mathcal{M}$ is in fact ample on all but finitely many fibers of $f$. After repeating the construction for each such fiber and forming the tensor product, we have constructed a $f$-relatively ample line bundle on $X$. This completes the proof.

We now turn our attention to the moduli spaces.

4.1. Esteves Jacobians. We first discuss the Esteves moduli space of quasistable sheaves. This moduli space fits very naturally into the framework of the previous section.

Suppose $B$ be a locally noetherian scheme and $f: X \rightarrow B$ a projective family of curves whose fibers are geometrically reduced. Quasistability is defined in terms of a section $\sigma: B \rightarrow X^{\mathrm{sm}}$ and a vector bundle $\mathscr{E}$ on $X$ with fiberwise integral slope $\operatorname{deg}\left(\mathscr{E}_{b}\right) / \operatorname{rank}\left(\mathscr{E}_{b}\right)$, which we assume is constant as a function of $b \in B$. Given $\sigma$ and $\mathscr{E}, \sigma$-quasistability is a numerical condition on the multidegree of a rank 1 , torsion-free sheaf of degree

$$
d(\mathscr{E})=d:=-\chi\left(\mathscr{O}_{X_{b}}\right)-\operatorname{deg}\left(\mathscr{E}_{b}\right) / \operatorname{rank}\left(\mathscr{E}_{b}\right) .
$$

For the definitions (which we will not use), we direct the reader to [Esteves 2001, p. 3051] (for a single sheaf) and [ibid., p. 3054] (for a family). The basic existence theorem is [ibid., Theorem A on p. 3047], which is proved in [ibid., Section 4]). It states that if $\mathrm{Sheaf}_{\mathscr{E}}^{\sigma}: S$-Sch $\rightarrow$ Sets is the functor defined by setting $\operatorname{Sheaf}_{\mathscr{E}}^{\sigma}(T)$ equal to the set of isomorphism classes of $O_{T}$-flat, finitely presented $\mathscr{O}_{X_{T}}$-modules that are fiberwise $\sigma$-quasistable, then there is a $B$-proper algebraic space $\bar{J}_{\mathscr{E}}^{\sigma} \rightarrow B$ of finite type that represents the étale sheaf associated to Sheaf ${ }_{\mathscr{c}}^{\sigma}$.

Strictly speaking, our definition differs from the one given in [ibid.] in two ways. First, Esteves does not work with isomorphism classes of sheaves but rather with equivalence classes under the relation given by identifying two sheaves $\Phi_{1}$ and $\mathscr{I}_{2}$ on $X_{T}$ when $\Phi_{1}$ is isomorphic to $\Phi_{2} \otimes f^{*}(\mathscr{L})$ for some line bundle $\mathscr{L}$ on $T$. Zariski locally on $T$, the sheaves $\Phi_{1}$ and $\Phi_{2}$ are isomorphic, and it follows that the étale sheaf associated to Sheaf $f_{\mathscr{E}}^{\sigma}$ is canonically isomorphic to the sheaf considered by Esteves. Second, Esteves only defines his functor as a functor from locally noetherian schemes to sets. However, the functor Sheaf $f_{\mathscr{E}}^{\sigma}$ and its associated étale sheaf are easily seen to be locally finitely presented. It follows that $\bar{J}_{\mathscr{E}}^{\sigma}$ represents the étale sheaf associated to Sheaf ${ }_{\mathscr{E}}^{\sigma}$, rather than just the restriction of this sheaf to locally noetherian schemes. 
If $f$ satisfies stronger conditions, then the space $\bar{J}_{\mathscr{E}}^{\sigma}$ is actually a scheme. This is the content of [Esteves 2001, Theorem B, p. 3048], proved on [ibid., p. 3086]. The theorem states that if there exist sections $\sigma_{1}, \ldots, \sigma_{n}: B \rightarrow X^{\mathrm{sm}}$ of $f$ with the property that every irreducible component of a fiber $X_{b}$ is geometrically integral and contains one of the points $\sigma_{1}(b), \ldots, \sigma_{n}(b)$, then $\bar{J}_{\mathscr{E}}^{\sigma}$ is a scheme.

In the special case where $B=S$ is a strict henselian discrete valuation ring with generic point $\eta$ and special point 0 , the hypotheses of Theorem B are automatically satisfied. Indeed, the locus of $k(0)$-valued points is dense in the smooth locus $X_{0}^{\mathrm{sm}}$ (Fact 3.4), which in turn is dense in $X_{0}$ as $X_{0}$ is geometrically reduced. We may conclude that the irreducible components of $X_{0}$ are geometrically integral. Finally, every $k(0)$-valued point of $X_{0}$ extends to a section $\sigma: S \rightarrow X$ (Fact 3.5), so the hypotheses of Theorem B are certainly satisfied.

We call $\bar{J}_{\mathscr{E}}^{\sigma}$ the Esteves compactified Jacobian. Inside of the Esteves compactified Jacobian, we can consider the open subscheme parametrizing line bundles. This scheme is called the Esteves Jacobian and denoted by $J_{\mathscr{E}}^{\sigma}$. While the scheme $\bar{J}_{\mathscr{E}}^{\sigma}$ parametrizes sheaves, it is not naturally a subfunctor of Sh because it does not parametrize degree 0 sheaves. We can, however, define a natural transformation $\bar{J}_{\mathscr{E}}^{\sigma} \rightarrow$ Sh by the rule

$$
\mathscr{I} \mapsto \mathscr{I}(-d \cdot \sigma)
$$

Both Proposition 3.6 and Theorem 3.9 apply to $\bar{J}_{\mathscr{E}}^{\sigma}$.

Corollary 4.2. Fix a Dedekind scheme $B$. Let $f: X \rightarrow B$ be a projective family of geometrically reduced curves. Let $\sigma: B \rightarrow X^{s m}$ be a section and $\mathscr{E}$ a vector bundle on $X$ with fiberwise integral slope.

Then the natural map $J_{\mathscr{E}}^{\sigma} \rightarrow \mathrm{Q}$ is an open immersion.

Assume further that $f$ satisfies Hypothesis 1 . Then $J_{\mathscr{E}}^{\sigma}=\mathrm{Q}^{\tau}$, and this scheme is the Néron model.

Proof. By localizing, we can assume that $B=S$ is a strict henselian discrete valuation ring, in which case we are reduced to proving that the hypotheses of Proposition 3.6 and Theorem 3.9 hold. The scheme $J_{\mathscr{E}}^{\sigma}$ is easily seen to be formally $S$-smooth. Indeed, $\sigma$-quasistability is a condition on fibers, so the formal smoothness of $\mathrm{P}^{0}$ implies the formal smoothness of $J_{\mathscr{c}}^{\sigma}$. The remaining hypotheses of Proposition 3.6 are explicitly assumed, so we can deduce the first part of the theorem. To complete the proof, it is enough to show that $J_{\mathscr{E}}^{\sigma}$ is stabilized by $\mathrm{Q}^{\mathrm{o}}$. But the action of $\mathrm{Q}^{\mathrm{o}}$ on $J_{\mathscr{E}}^{\sigma}$ is given by the tensor product against a multidegree 0 line bundle, so this action preserves multidegree and hence $\sigma$-quasistability.

Corollary 4.2 implies that $J_{\mathscr{g}}^{\sigma}$ is a scheme with (unique) $B$-group scheme structure that extends the group scheme structure of the generic fiber. It is not immediate from the definition that $J_{\mathscr{E}}^{\sigma}$ admits such structure, and Example 4.9 shows that the 
group structure is special to the case of families over a 1-dimensional base. The result also implies uniqueness results for the Esteves Jacobian; if $\sigma^{\prime}: B \rightarrow X^{\mathrm{sm}}$ is a second section and $\mathscr{E}^{\prime}$ a second vector bundle on $X$, then $J_{\mathscr{E}}^{\sigma^{\prime}}$ is canonically isomorphic to $J_{\mathscr{G}}^{\sigma}$. In the next section, we will define the Simpson stable Jacobian $J_{\mathscr{L}}^{0}(X)$, and this scheme is also isomorphic to $J_{\mathscr{E}}^{\sigma}$ provided every slope semistable sheaf is stable. It would be interesting to know if these isomorphisms extend to the compactifications. Important results along these lines can be found in [Melo and Viviani 2012; Esteves 2009], but many basic question remain unanswered. Currently, there is no example of a curve $X_{0} \rightarrow \operatorname{Spec}(k)$ such that two Esteves compactified Jacobians associated to $X_{0}$ are nonisomorphic.

4.2. Simpson Jacobians. The hypotheses to Proposition 3.6 and Theorem 3.9 are satisfied by certain moduli spaces of stable sheaves, which we call Simpson Jacobians. Here we recall Simpson's construction, along with later work of Langer and Maruyama, and then apply results from Section 3. We restrict our attention to families of reduced curves (but see Remark 4.4, and the discussion preceding Example 4.9).

We work over a scheme $B$ that is finitely generated over a universally Japanese ring $R$ (e.g., $R=\mathbb{C}, \mathbb{F}_{p}, \mathbb{Z}, \ldots$ ). Let $f: X \rightarrow B$ a family of curves with $f$-relatively ample line bundle $\mathscr{L}$, and assume the Euler-Poincaré characteristics $\chi\left(0_{X_{b}}\right)$ and $\chi\left(\mathscr{L}_{b}\right)$ are constant as functions of the base $B$. Set $P_{d}$ equal to the polynomial

$$
P_{d}(t):=\operatorname{deg}\left(\mathscr{L}_{b}\right) \cdot t+d+\chi,
$$

where $\chi$ is the Euler-Poincaré characteristic of a fiber of $f$ and $\operatorname{deg}\left(\mathscr{L}_{b}\right)$ is the degree of the restriction of $\mathscr{L}$ to a fiber. This is the Hilbert polynomial of a degree $d$ line bundle.

Given this data, Simpson constructed an associated moduli space in the case that $R=\mathbb{C}$. The Simpson moduli space $\mathrm{M}\left(0_{X}, P_{d}\right)$ parametrizes slope semistable sheaves with Hilbert polynomial $P_{d}$. (See [Simpson 1994, pp. 54-56] for the definition of semistability). To be precise, define $\mathrm{M}^{\sharp}\left(\mathscr{O}_{X}, P_{d}\right)$ to be the functor whose $T$-valued points are isomorphism classes of $0_{T}$-flat, finitely presented $0_{X_{T}}$ modules whose fibers are $\mathscr{L}$-slope semistable sheaves with Hilbert polynomial $P_{d}$. The main existence result [Simpson 1994, Theorem 1.21] asserts that there is a projective scheme $\mathrm{M}\left(\mathrm{O}_{X}, P_{d}\right)$ that corepresents $\mathrm{M}^{\sharp}\left(\mathrm{O}_{X}, P_{d}\right)$. Inside of $\mathrm{M}\left(\mathrm{O}_{X}, P_{d}\right)$, we may consider the open subscheme $\mathrm{M}^{\mathrm{st}}\left(\mathrm{O}_{X}, P_{d}\right)$ parametrizing $\mathscr{L}$-slope stable sheaves. The stable locus is a fine moduli space: Its $\mathbb{C}$-valued points are in natural bijection with the isomorphism classes of $\mathscr{L}$-slope stable sheaves with Hilbert polynomial $P_{d}$, and étale locally on $\mathrm{M}^{\mathrm{st}}\left(\mathrm{O}_{X}, P_{d}\right)$, the product $X \times{ }_{B} \mathrm{M}^{\mathrm{st}}\left(\mathrm{O}_{X}, P_{d}\right)$ admits a universal family of sheaves. The reader may check that these conditions 
are equivalent to the condition that $\mathrm{M}^{\mathrm{st}}\left(\mathrm{O}_{X}, P_{d}\right)$ represents the étale sheaf associated the functor parametrizing stable sheaves. While Simpson only considers the case $R=\mathbb{C}$, later work of Langer [2004a, Theorem 4.1; 2004b, Theorem 0.2] and Maruyama [1996] extends these results to the case where $R$ is an arbitrary universally Japanese ring.

Let us now specialize to the case where $B$ is a Dedekind scheme. When $f$ has reducible fibers, $\mathrm{M}^{\mathrm{st}}\left(\mathrm{O}_{X}, P_{d}\right)$ may contain points corresponding to sheaves that are not rank 1; see [López-Martín 2005, Example 2.2]. This is potentially a major source of confusion: The term "rank" is used in a different way in [Simpson 1994], and the sheaves parametrized by $\mathrm{M}^{\mathrm{st}}\left(\mathrm{O}_{X}, P_{d}\right)$ are rank 1 in Simpson's sense but not necessary in the sense used here.

We avoid these sheaves. Define the Simpson stable Jacobian $J_{\mathscr{L}}^{d}$ of degree $d$ to be the locus of stable line bundles in $\mathrm{M}^{\mathrm{st}}\left(\mathrm{O}_{X}, P_{d}\right)$ (which is an open subscheme by [Altman and Kleiman 1980, Lemma 5.12(a)]). We define the Simpson stable compactified Jacobian $\bar{J}_{\mathscr{L}}^{d}$ to be the subset of the stable locus $\mathrm{M}^{\mathrm{st}}\left(\mathrm{O}_{X}, P_{d}\right)$ that corresponds to pure, rank 1 sheaves. (Warning: The compactified Jacobian is a $B$-proper scheme when every semistable pure sheaf with Hilbert polynomial $P_{d}$ is stable but not in general!)

When the fibers of $X \rightarrow B$ are geometrically reduced, a minor modification of the proof of [Pandharipande 1996, Lemma 8.1.1] shows that the subset $\bar{J}_{\mathscr{L}}^{d} \subset$ $\mathrm{M}^{\mathrm{st}}\left(\mathrm{O}_{X}, P_{d}\right)$ is closed and open, and hence has a natural scheme structure:

Lemma 4.3. Assume the fibers of $f: X \rightarrow B$ are geometrically reduced. Then the subset $\bar{J}_{\mathscr{L}}^{d}$ is closed and open in $\mathrm{M}^{s t}\left(\mathcal{O}_{X}, P_{d}\right)$.

Proof. The main point to prove is that a 1-parameter family of line bundles cannot specialize to a pure sheaf that fails to have rank 1 , and this is shown by examining the leading term of the Hilbert polynomial. To begin, we may cover $\mathrm{M}^{\mathrm{st}}\left(\mathrm{O}_{X}, P_{d}\right)$ by étale morphisms $\mathrm{M} \rightarrow \mathrm{M}^{\mathrm{st}}\left(\mathrm{O}_{X}, P_{d}\right)$ with the property that a universal family $\Phi_{\text {uni. }}$. on $\mathrm{M} \times{ }_{B} X$ exists. It is enough to verify the claim after passing from $\mathrm{M}^{\mathrm{st}}\left(\mathrm{O}_{X}, P_{d}\right)$ to an arbitrary such scheme, and so for the remainder of the proof we work with $\mathrm{M}$ in place of $\mathrm{M}^{\mathrm{st}}\left(\mathrm{O}_{X}, P_{d}\right)$. We will also abuse notation by denoting the pullback of $\bar{J}_{\mathscr{L}}^{d}$ under $\mathrm{M} \rightarrow \mathrm{M}^{\mathrm{st}}\left(\mathrm{O}_{X}, P_{d}\right)$ by the same symbol $\bar{J}_{\mathscr{L}}^{d}$.

We first need to check that $\bar{J}_{\mathscr{L}}^{d} \subset \mathrm{M}$ is constructible, so that we can make use of the valuative criteria. Given $m \in M$ mapping to $b \in B$, the condition that the fiber $I_{m}$ is rank 1 is just the condition that the restriction of $I_{m}$ to $X_{b}^{\mathrm{sm}}$ is a line bundle. Constructibility thus follows from [Grothendieck 1966a, 9.4.7] applied to $\mathrm{M} \times{ }_{B} X^{\mathrm{sm}} \rightarrow \mathrm{M}$.

To finish, it is enough to prove that $\bar{J}_{\mathscr{L}}^{d}$ is closed under specialization and generalization. Thus, we pass from $\mathrm{M}$ to a discrete valuation ring $T$ mapping to $\mathrm{M}$. If 
$\mathscr{I}$ is the sheaf on $X_{T}$ given by the pullback of the universal family, then we need to show that the generic fiber of $I_{\eta}$ is rank 1 if and only if the special fiber $I_{0}$ is.

To prove this, we turn our attention to the Hilbert polynomial $P_{d}$ of a fiber of $I$. This polynomial is defined so that the leading term is $\operatorname{deg}\left(\mathscr{L}_{b}\right)$, and we can express this number in terms of components of a fiber of $X_{T} \rightarrow T$ as follows. If $x$ is generic point of the special fiber $X_{0}$, then we define $\operatorname{deg}_{x}\left(\mathscr{L}_{0}\right)$ to be the degree of the restriction of $\mathscr{L}_{0}$ to the irreducible component corresponding to $x$. (Give the component the reduced subscheme structure.) For any generic point $y$ of $X_{\eta}$, we define $\operatorname{deg}_{y}\left(\mathscr{L}_{\eta}\right)$ in the analogous manner. If $x_{1}, \ldots, x_{n}$ are all the generic points of $X_{0}$ and $y_{1}, \ldots, y_{m}$ all the generic points of $X_{\eta}$, then we have

$$
\begin{aligned}
\operatorname{deg}\left(\mathscr{L}_{b}\right) & =\operatorname{deg}_{x_{1}}\left(\mathscr{L}_{0}\right)+\cdots+\operatorname{deg}_{x_{n}}\left(\mathscr{L}_{0}\right) \\
& =\operatorname{deg}_{y_{1}}\left(\mathscr{L}_{\eta}\right)+\cdots+\operatorname{deg}_{y_{m}}\left(\mathscr{L}_{\eta}\right)
\end{aligned}
$$

by, say, [Altman and Kleiman 1979, 2.5.1]. The terms $\operatorname{deg}_{x_{i}}\left(\mathscr{L}_{0}\right)$ and $\operatorname{deg}_{y_{j}}\left(\mathscr{L}_{\eta}\right)$ in the equation above are each strictly positive as $\mathscr{L}$ is relatively ample.

We can also express $\operatorname{deg}\left(\mathscr{L}_{b}\right)$ in terms of the generic rank of a fiber of $I$. Using [Altman and Kleiman 1979, 2.5.1] again, we have

$$
\begin{aligned}
\operatorname{deg}\left(\mathscr{L}_{b}\right) & =\operatorname{deg}_{x_{1}}\left(\mathscr{L}_{0}\right) \cdot \ell_{x_{1}}\left(I_{0}\right)+\cdots+\operatorname{deg}_{x_{n}}\left(\mathscr{L}_{0}\right) \cdot \ell_{x_{n}}\left(I_{0}\right) \\
& =\operatorname{deg}_{y_{1}}\left(\mathscr{L}_{\eta}\right) \cdot \ell_{y_{1}}\left(I_{\eta}\right)+\cdots+\operatorname{deg}_{y_{m}}\left(\mathscr{L}_{\eta}\right) \cdot \ell_{y_{m}}\left(I_{\eta}\right) .
\end{aligned}
$$

Here $\ell_{x_{i}}\left(I_{0}\right)$ denotes the length of the localization of $I_{0}$ at $x_{i}$ and similarly for $\ell_{y_{j}}\left(I_{\eta}\right)$. The fibers of $X_{T} \rightarrow T$ are reduced, so such a length is equal to the minimal number of generators. In particular, these numbers are upper semicontinuous. In other words, if $y_{j}$ specializes to $x_{i}$, then we have $\ell_{y_{j}}\left(I_{\eta}\right) \leq \ell_{x_{i}}\left(I_{0}\right)$ (by Nakayama's lemma).

The desired result now follows. Suppose first that $I_{0}$ is rank 1 . Then we have $\ell_{y_{i}}\left(I_{\eta}\right) \leq 1$ for all $i$ by semicontinuity. If some inequality was strict, say $\ell_{y_{1}}\left(I_{\eta}\right)=0$, then we would have

$$
\begin{aligned}
\operatorname{deg}\left(\mathscr{L}_{b}\right) & =\operatorname{deg}_{y_{1}}\left(\mathscr{L}_{\eta}\right)+\operatorname{deg}_{y_{2}}\left(\mathscr{L}_{\eta}\right)+\cdots+\operatorname{deg}_{y_{m}}\left(\mathscr{L}_{\eta}\right) \\
& >\quad \operatorname{deg}_{y_{2}}\left(\mathscr{L}_{\eta}\right)+\cdots+\operatorname{deg}_{y_{m}}\left(\mathscr{L}_{\eta}\right) \\
& \geq \operatorname{deg}_{y_{1}}\left(\mathscr{L}_{\eta}\right) \cdot \ell_{y_{1}}\left(I_{\eta}\right)+\cdots+\operatorname{deg}_{y_{m}}\left(\mathscr{L}_{\eta}\right) \cdot \ell_{y_{m}}\left(I_{\eta}\right) \\
& =\operatorname{deg}\left(\mathscr{L}_{b}\right) .
\end{aligned}
$$

This is absurd! Thus, we must have $\ell_{y_{i}}\left(I_{\eta}\right)=1$ for all $y_{i}$ and $I_{\eta}$ is rank 1 . Similar reasoning shows that if $I_{\eta}$ is rank 1 , then $I_{0}$ is rank 1 .

Remark 4.4. The hypothesis that the fibers of $f$ are geometrically reduced is necessary. Indeed, the moduli space $\mathrm{M}^{\mathrm{st}}\left(\mathrm{O}_{X}, P_{d}\right)$ was described in [Chen and Kass 2011] in the case that $X$ is a nonreduced curve whose reduced subscheme $X_{\text {red }}$ 
is smooth and whose nilradical $\mathcal{N}$ is square-zero (i.e., $X$ is a ribbon). Using that description it is easy to produce examples where $\bar{J}_{\mathscr{L}}^{d} \subset \mathrm{M}^{\text {st }}\left(\mathrm{O}_{X}, P_{d}\right)$ is not closed (e.g., take $d$ equal to $0, X$ to have even genus, and $X_{\text {red }}$ to have genus 1 ). The points of the complement in the closure correspond to stable rank 2 vector bundles on $X_{\text {red }}$.

We now apply Proposition 3.6 and Theorem 3.9 to the Simpson Jacobians.

Corollary 4.5. Fix a Dedekind scheme $B$ that is finitely generated over a universally Japanese ring. Let $f: X \rightarrow B$ be a family of geometrically reduced curves. Let $\mathscr{L}$ be $f$-relatively ample line bundle.

Then the natural map $J_{\mathscr{L}}^{0}(X) \rightarrow \mathrm{Q}^{\tau}$ is an open immersion. Assume further that both of the following conditions hold:

- Every $\mathscr{L}$-slope semistable rank 1 , torsion free sheaf of degree 0 is $\mathscr{L}$-slope stable.

- $f$ satisfies Hypothesis 1.

Then $J_{\mathscr{L}}^{0}(X)=\mathrm{Q}^{\tau}$, and this scheme is the Néron model.

Proof. The local existence of a universal family [Simpson 1994, Theorem 2.1(4)] implies that there is a natural transformation $\bar{J}_{\mathscr{L}}(X) \rightarrow$ Sh with the property that $J_{\mathscr{L}}(X)$ is the preimage of $\mathrm{P}^{0} \subset \mathrm{Sh}$. Furthermore, the slope stability condition is a fiberwise condition, so a modification of the argument given in Corollary 4.2 completes the proof.

Remark 4.6. A minor generalization of Corollary 4.5 can be obtained by allowing for moduli spaces of degree $d$ lines bundles, with $d \neq 0$. If we are given a line bundle $\mathcal{M}$ on $X$ with fiberwise degree $d$, then there is an associated map $J_{\mathscr{L}}^{d}(X) \rightarrow \mathrm{Q}$ that extends the map on the generic fiber given by tensoring with $\mathcal{M}^{-1}$. With only notational changes the previous corollary generalizes to a statement about this map.

Corollary 4.5 is, of course, only of interest when there exists an $\mathscr{L}$ such that $\mathscr{L}$-slope stability coincides with $\mathscr{L}$-slope semistability. Thus, we ask, When does such an $\mathscr{L}$ exist? A comprehensive discussion of this question would require a digression on stability conditions, so we limit ourselves to reviewing known results about a single curve $X_{0}$ over an algebraically closed field. When $X_{0}$ is integral, the stability condition is vacuous, so every ample $\mathscr{L}_{0}$ has the desired property. If $X_{0}$ is reducible of genus $g \neq 1$ with only nodes as singularities, then Melo and Viviani have proven the existence of a suitable $\mathscr{L}_{0}$ [2012, Proposition 6.4]. Stability conditions on reduced, genus 1 curves were analyzed by López-Martín [2005]. She exhibits curves $X_{0}$ with the property that there is no $\mathscr{L}_{0}$ such that every $\mathscr{L}_{0}$-slope semistable, pure, rank 1 sheaf degree 0 is stable, but a suitable $\mathscr{L}_{0}$ always exists if one considers sheaves of fixed degree $d \neq 0$. Finally, stability conditions for a 
ribbon were analyzed in [Chen and Kass 2011]. On a ribbon, the stability condition is independent of $\mathscr{L}_{0}$, and for rational ribbons, slope stability coincides with slope semistability precisely when the genus $g$ is even. It would be desirable to have a general result asserting (non)existence of a suitable $\mathscr{L}_{0}$.

4.3. Genus 1 curves. The Néron model of the Jacobian of a genus 1 curve can be quite complicated (see for example [Liu et al. 2004]), but these complications do not arise if the family admits a section. Suppose $B$ is a Dedekind scheme and $f: X \rightarrow B$ is a family of curves such that the total space $X$ is regular and the generic fiber $X_{\eta}$ is smooth. If $\sigma: B \rightarrow X^{\mathrm{sm}}$ is a section contained in the smooth locus, then there is a canonical identification of the smooth locus $X^{\text {sm }}$ with the Néron model $\mathrm{N}$ of the Jacobian of $X_{\eta}$. Here we examine how this fact fits into the preceding framework.

Definition 4.7. Let $f: X \rightarrow B$ be a family of genus 1 curves over a Dedekind scheme and $\sigma: B \rightarrow X^{\text {sm }}$ a section contained in the smooth locus. We define a sheaf $\Phi_{\text {uni. }}$ on $X \times{ }_{B} X$ by the formula

$$
\mathscr{I}_{\text {uni. }}:=\mathscr{I}_{\Delta}\left(\pi_{1}^{*}(\sigma)+\pi_{2}^{*}(\sigma)\right) .
$$

Here $\Phi_{\Delta}$ is the ideal sheaf of the diagonal, and $\pi_{1}, \pi_{2}: X \times_{B} X \rightarrow X$ are the projection maps.

The sheaf $\Phi_{\text {uni. }}$ determines a transformation $X \rightarrow$ Sh that realizes $X$ as a moduli space of sheaves over itself. Proposition 3.6 and Theorem 3.9 apply to this moduli space.

Corollary 4.8. Fix a Dedekind scheme B. Let $f: X \rightarrow B$ be a family of genus 1 curves. Let $\sigma: B \rightarrow X^{\mathrm{sm}}$ be a section.

Then the natural map $X^{\mathrm{sm}} \rightarrow \mathrm{Q}$ is an open immersion.

Assume further that $f$ satisfies Hypothesis 1. Then $X^{\mathrm{sm}}=\mathrm{Q}^{\tau}$, and this scheme is the Néron model.

Let us consider the special case where $B$ is a discrete valuation ring, $X$ is a minimal regular surface, and the residue field $k(0)$ is algebraically closed. The possibilities for the special fiber $X_{0}$ are given by the Kodaria-Néron classification ([Kodaira 1960; Néron 1964]; see [Silverman 1994, pp. 353-354] for a recent exposition). The reduced curves appearing in the classification are the reduction types $\mathrm{I}_{n}$, II, III, and IV. In these cases, one may show that the induced morphism $X \rightarrow$ Sh identifies $X$ with the Esteves compactified Jacobian $\bar{J}_{\mathscr{O}}^{\sigma}$.

In every remaining case (reduction type $\mathrm{I}_{n}^{*}, \mathrm{II}^{*}, \mathrm{III}^{*}$, or $\mathrm{IV}^{*}$ ) the morphism $X \rightarrow$ Sh is not a special case of the fine moduli spaces discussed in the previous two sections. Indeed, the special fiber $X_{0}$ is nonreduced, so the Esteves Jacobian of $X$ is not defined. In Section 4.2, we reviewed Simpson's moduli space $\mathrm{M}^{\mathrm{st}}\left(\mathcal{O}_{X}, P_{d}\right)$ of 
stable sheaves, but the image of $X \rightarrow$ Sh cannot be described as a closed subscheme of that space. The reason is that slope stable sheaves are simple, but some fibers of $\mathscr{I}_{\text {uni. }}$ are not simple. Specifically, if $p_{0} \in X_{0}$ lies on the intersection of two components, then the fiber of $\mathscr{I}_{\text {uni }}$ of $p_{0}$ fails to be simple. This can be seen as follows. This fiber is the sheaf $\mathscr{I}_{p_{0}}(+\sigma(0))$, where $\mathscr{I}_{p_{0}}$ is the ideal of $p_{0}$. If $v: X_{0}^{\prime} \rightarrow X_{0}$ is the blow-up of $X_{0}$ at $p_{0}$, then one may show that $H^{0}\left(X_{0}^{\prime}, O_{X_{0}^{\prime}}\right)$ is canonically isomorphic to the endomorphism ring of $\Phi_{p_{0}}(+\sigma(0))$. An inspection of the Kodaria-Néron table shows that $X_{0}^{\prime}$ is disconnected, so $H^{0}\left(X_{0}^{\prime}, O_{X_{0}^{\prime}}\right)$ does not equal $k(0)$ and $\mathscr{I}_{p_{0}}(+\sigma(0))$ is not simple.

Corollary 4.8 provides a partial answer to a question posed in the introduction: What are the maximal subfunctors $J$ of $\mathrm{P}^{0}$ represented by a separated $B$-scheme? When $X$ is, say, regular, a strong result one could hope for is that there is always a subfunctor $\bar{J}$ of Sh satisfying the hypotheses of Theorem 3.9. The line bundle locus $J \subset \bar{J}$ in such a functor has the property that $J \rightarrow \mathrm{Q}^{\tau}$ is an isomorphism, and hence $J$ is maximal. Corollary 4.8 shows that such a $\bar{J}$ exists when $f: X \rightarrow S$ is a family of genus 1 curves that $f$ admits a section. Similarly, the Esteves compactified Jacobian represents a suitable subfunctor when $f$ has geometrically reduced fibers and admits a section. In general, however, the hope is too optimistic: Raynaud's family, mentioned at the end of Section 3, has that property that no such $\bar{J}$ can exist.

The question of describing maximal subfunctors $J$ is most interesting when $f$ has nonreduced fibers. The slope stable line bundles form a subfunctor $J \subset \mathrm{P}^{0}$ represented by a $S$-separated scheme, but our discussion of genus 1 families together with Remark 4.4 suggest that we should consider other methods for constructing a suitable $J$ when $f$ has nonreduced fibers.

In a different direction, one nice property of the moduli spaces described by Corollary 4.8 is that their geometry is very simple. We use these spaces to provide an example showing that a family $J \rightarrow B$ of Esteves Jacobians over a regular 2-dimensional base may not have group scheme structure.

Example 4.9 (Néron models in 2-dimensional families). We will construct a 2dimensional family $f: X \rightarrow B$ of plane cubics and an associated Esteves Jacobian $J \rightarrow B$ with the property that the group law on the locus $J_{U} \rightarrow U$ parametrizing nonsingular cubics does not extend over all of $B$. Furthermore, the family is constructed so that a dense open subset of $B$ is covered by nonsingular curves $C$ with the property that the restriction $X_{C}$ of $X$ to $C$ is regular, so $J_{C}$ is the Néron model of its generic fiber (and in particular admits group scheme structure that extends the group scheme structure over $C \cap U$ ). Thus, the Néron models fit into a 2-dimensional family, but their group scheme structure does not.

The idea is as follows. The family we construct has a reducible element $X_{b_{0}} \rightarrow$ $b_{0}$ with the property that, for every nonsingular curve $C \subset B$ passing through $b_{0}$ 
such that $X_{C}$ is regular, the restriction of the Esteves Jacobian $J_{C}$ is the Néron model of its generic fiber. The fiber $J_{b_{0}}$ inherits a group law from this Néron model, and we show explicitly that this group law depends on the particular choice of $C$. But, if the group law on $J_{U}$ extended to $J$, then all the different group laws on $J_{b_{0}}$ coming from the different curves $C$ would be the restriction of one common group law on $J$, which is absurd. We now construct the family.

We work over an algebraically closed field $k$. The family $X \rightarrow B$ will be a net of plane cubics. Let $X_{0} \subset \mathbb{P}_{k}^{2}$ be a reducible plane cubics that is the union of a smooth quadric $Q_{0}$ and a line $L_{0}$ that meet in two distinct points. (See Figure 1.) Fix two general points $p_{1}, p_{2} \in L_{0}(k)$ on the line and one general point $q_{1} \in Q_{0}(k)$ on the quadric. Say that $F \in H^{0}\left(\mathbb{P}_{k}^{2}, \mathcal{O}(3)\right)$ is an equation for $X_{0}$ and $G, H \in H^{0}\left(\mathbb{P}_{k}^{2}, \mathcal{O}(3)\right)$ are two general cubic equations that vanish on all of the points $p_{1}, p_{2}, q_{1}$. We will work with the net $V:=\langle F, G, H\rangle \subset H^{0}\left(\mathbb{P}_{k}^{2}, \mathcal{O}(3)\right)$ and the associated family of curves

$$
\begin{aligned}
X & :=\{(p,[r, s, t]): r \cdot F(p)+s \cdot G(p)+t \cdot H(p)=0\} \\
& \subset \mathbb{P}_{k}^{2} \times \mathbb{P}_{k}^{2} .
\end{aligned}
$$

There are two obvious morphisms $e, f: X \rightarrow \mathbb{P}_{k}^{2}$ given by the two projections. If we set $B:=\mathbb{P}_{k}^{2}$ equal to the plane, then the second morphism $f: X \rightarrow B$ realizes $X$ as a family of genus 1 curves with $X_{0}=f^{-1}\left(b_{0}\right)$, where $b_{0}:=[1,0,0]$. Corresponding to the points $p_{1}, p_{2}, q_{1} \in X_{0}(k)$ are three section $\sigma_{1}, \sigma_{2}, \tau_{1}: B \rightarrow X^{\text {sm }}$, which lie in the smooth locus by the generality assumption.

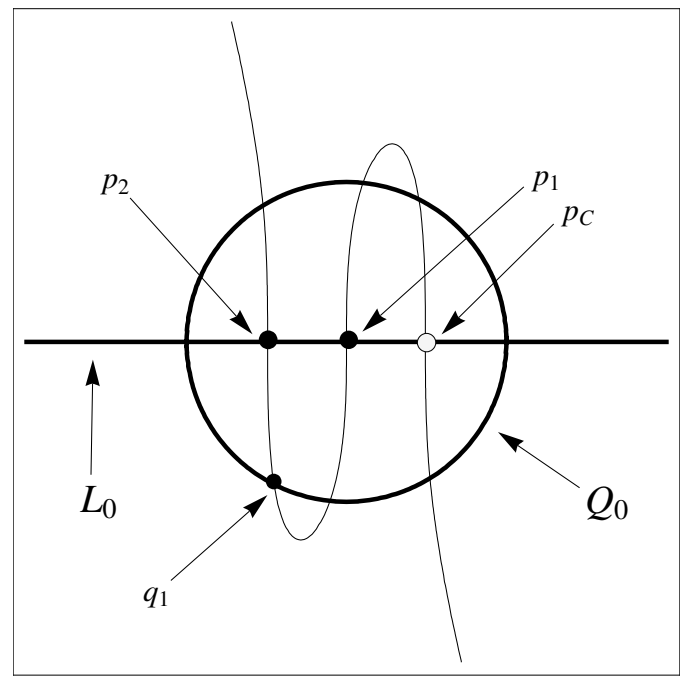

Figure 1. The pencil $X_{C}$. 
Another application of the generality assumption shows that the fibers of $f$ are reduced, so we can form the Esteves Jacobian $J:=J_{\mathscr{E}}^{\sigma_{1}}$, where $\mathscr{E}=\mathscr{O}_{X}$. The quasistability condition on a line bundle $\mathscr{L}_{0}$ on $X_{0}$ is the condition that the bidegree $\left(\operatorname{deg}\left(\mathscr{L}_{L_{0}}\right), \operatorname{deg}\left(\mathscr{L}_{Q_{0}}\right)\right)$ equals $(0,0)$ or $(1,-1)$. Now we assume $J \rightarrow B$ is a group scheme and derive a contradiction.

Suppose that we are given a general line $C \subset B$ in the plane that contains $b_{0}$. Such a line corresponds to a 2-dimensional linear subspace of the form $W:=$ $\left\langle F, G_{C}\right\rangle \subset V$ for some $G_{C} \in V$. Invoking generality again, the base locus

$$
\left\{p \in \mathbb{P}_{k}^{2}: F(p)=G_{C}(p)=0\right\}
$$

consists of 9 distinct points. The first projection map $e: X \rightarrow \mathbb{P}_{k}^{2}$ realizes $X_{C}$ as the blow-up of the plane at these points, so $X_{C}$ is regular, and thus $J_{C}$ is the Néron model of its generic fiber. We now study the group of sections of $J_{C} \rightarrow C$.

The base locus (4-4) includes the points $p_{1}, p_{2}, q_{1}$. In addition to the points $p_{1}, p_{2}$, a unique third point of the base locus must lie on the line $L_{0}$. Let us label that point $p_{C}$ and write $\sigma_{C}: C \rightarrow X_{C}$ for the corresponding section.

Now consider the following line bundles on $X_{C}$ :

$$
\begin{aligned}
\mathscr{L}_{1}:=\mathcal{O}\left(\sigma_{1}-\tau_{1}\right), & \mathscr{L}_{C}:=\mathcal{O}\left(\sigma_{C}-\tau_{1}\right), \\
\mathscr{L}_{2}:=\mathcal{O}\left(\sigma_{2}-\tau_{1}\right), & \mathcal{M}:=\mathcal{O}(1) \otimes \mathcal{O}\left(-3 \cdot \tau_{1}\right) .
\end{aligned}
$$

These lines bundles are all $\sigma_{1}$-quasistable. If we let $g_{1}, g_{2}, g_{C}, h \in J_{C}(C)$ respectively correspond to $\mathscr{L}_{1}, \mathscr{L}_{2}, \mathscr{L}_{C}, \mu$, then I claim we have

$$
g_{1}+g_{2}+g_{C}=h
$$

Indeed, it is enough to verify the claim after passing to the generic fiber of $J_{C} \rightarrow C$, where the equation is just the statement that the points $p_{1}, p_{2}, p_{C}$ all lie on a line (the line $L_{0}$ ). Now suppose that $J \rightarrow \mathbb{P}_{k}^{2}$ admits a group law extending the group law of the generic fiber. Then the specialization of (4-5) to $J_{b_{0}}$ holds for all $C$ simultaneously. In particular, the isomorphism class of the line bundle ${ }_{X_{b_{0}}}\left(p_{C}-q_{1}\right)$ is independent of the particular line $C \subset \mathbb{P}_{k}^{2}$ chosen. But this is absurd: For distinct general lines $C_{1}, C_{2}$, the points $p_{C_{1}}$ and $p_{C_{2}}$ (and hence the associated line bundles) are distinct! This completes our discussion of this example.

This example is particularly interesting in light of [Oda and Seshadri 1979]. The authors of that paper consider the case of a family of nodal curves $f: X \rightarrow B$ over a suitable Dedekind scheme with the property that $X$ is regular. Let $J_{\eta}$ be the Jacobian of the generic fiber. Given a closed point $0 \in B$, they prove that the special fiber $\mathrm{N}_{0}$ of the Néron model of $J_{\eta}$ depends only on the curve $X_{0}$ and not the particular family $f$ [ibid., Corollary 14.4]. This result must be interpreted with 
care: In our example, the group law depends on a particular choice of family, but any two such group laws define isomorphic group schemes.

\section{Acknowledgements}

The results of this paper are a part of my thesis. I would like to thank my advisor Joe Harris for his invaluable help. The thesis was carefully read by Filippo Viviani and Margarida Melo, who where very helpful in writing this paper, and I thank them. I thank Steven Kleiman for explaining the proof of Proposition 4.1, and Valery Alexeev for a useful discussion of stability conditions. I also thank the anonymous referee, Bryden Cais, Lucia Caporaso, Eduardo Esteves, Robert Lazarsfeld, and Dino Lorenzini for feedback about early drafts of this paper. Finally, I thank Bhargav Bhatt, Matthew Satriano, and Karen Smith for conversations that were helpful in clarifying technical aspects of this paper.

\section{References}

[Alexeev 2004] V. Alexeev, "Compactified Jacobians and Torelli map", Publ. Res. Inst. Math. Sci. 40:4 (2004), 1241-1265. MR 2006a:14016 Zbl 1079.14019

[Altman and Kleiman 1979] A. B. Altman and S. L. Kleiman, "Compactifying the Picard scheme, II”, Amer. J. Math. 101:1 (1979), 10-41. MR 81f:14025b Zbl 0427.14016

[Altman and Kleiman 1980] A. B. Altman and S. L. Kleiman, "Compactifying the Picard scheme", Adv. in Math. 35:1 (1980), 50-112. MR 81f:14025a Zbl 0427.14015

[Bosch et al. 1990] S. Bosch, W. Lütkebohmert, and M. Raynaud, Néron models, Ergeb. Math. Grenzgeb. (3) 21, Springer, Berlin, 1990. MR 91i:14034 Zbl 0705.14001

[Busonero 2008] S. Busonero, Compactified Picard schemes and Abel maps for singular curves, thesis, Università di Roma La Sapienz, 2008.

[Caporaso 1994] L. Caporaso, "A compactification of the universal Picard variety over the moduli space of stable curves”, J. Amer. Math. Soc. 7:3 (1994), 589-660. MR 95d:14014 Zbl 0827.14014

[Caporaso 2008a] L. Caporaso, "Compactified Jacobians, Abel maps and theta divisors", pp. 1-23 in Curves and abelian varieties (Athens, GA, 2007), edited by V. Alexeev et al., Contemp. Math. 465, Amer. Math. Soc., Providence, RI, 2008. MR 2010b:14088 Zbl 1152.14027

[Caporaso 2008b] L. Caporaso, "Néron models and compactified Picard schemes over the moduli stack of stable curves”, Amer. J. Math. 130:1 (2008), 1-47. MR 2009j:14030 Zbl 1155.14023

[Caporaso 2012] L. Caporaso, "Compactified Jacobians of Néron type", Rend. Lincei Mat. Appl. 23:2 (2012), 213-227. Zbl 1244.14031

[Casalaina-Martin et al. 2011] S. Casalaina-Martin, J. L. Kass, and F. Viviani, "The local structure of compactified Jacobians: Deformation theory", preprint, 2011. arXiv 1107.4166v1

[Chen and Kass 2011] D. Chen and J. L. Kass, "Moduli of generalized line bundles on a ribbon", preprint, 2011. arXiv 1106.5441v1

[D’Souza 1979] C. D'Souza, "Compactification of generalised Jacobians", Proc. Indian Acad. Sci. Sect. A Math. Sci. 88:5 (1979), 419-457. MR 81h:14004 Zbl 0442.14016

[Esteves 2001] E. Esteves, "Compactifying the relative Jacobian over families of reduced curves", Trans. Amer. Math. Soc. 353:8 (2001), 3045-3095. MR 2003b:14036 Zbl 0974.14009 
[Esteves 2009] E. Esteves, "Compactified Jacobians of curves with spine decompositions", Geom. Dedicata 139 (2009), 167-181. MR 2010d:14041 Zbl 1171.14018

[Gross 1990] B. H. Gross, "A tameness criterion for Galois representations associated to modular forms $(\bmod p)$ ), Duke Math. J. 61:2 (1990), 445-517. MR 91i:11060 Zbl 0743.11030

[Grothendieck 1961] A. Grothendieck, "Éléments de géométrie algébrique, III: Étude cohomologique des faisceaux cohérents, I", Inst. Hautes Études Sci. Publ. Math. 11 (1961), 5-167. MR 36 \#177c Zbl 0118.36206

[Grothendieck 1964] A. Grothendieck, "Éléments de géométrie algébrique, IV: Étude locale des schémas et des morphismes de schémas, I", Inst. Hautes Études Sci. Publ. Math. 20 (1964), 5-259. MR 30 \#3885 Zbl 0136.15901

[Grothendieck 1965] A. Grothendieck, "Éléments de géométrie algébrique, IV: Étude locale des schémas et des morphismes de schémas, II", Inst. Hautes Études Sci. Publ. Math. 24 (1965), 5231. MR 33 \#7330 Zbl 0135.39701

[Grothendieck 1966a] A. Grothendieck, "Éléments de géométrie algébrique, IV: Étude locale des schémas et des morphismes de schémas, III", Inst. Hautes Études Sci. Publ. Math. 28 (1966), 5255. MR 36 \#178 Zbl 0144.19904

[Grothendieck 1966b] A. Grothendieck, "Technique de descente et théorèmes d'existence en géométrie algébrique, VI, Les schémas de Picard: propriétés générales", exposé 236 in Séminaire Bourbaki 1961-1962, W. A. Benjamin, Amsterdam, 1966. Reprinted as pp. 221-243 in Séminaire Bourbaki 7, Soc. Math. France, Paris, 1995. MR 1610872 Zbl 0238.14015

[Grothendieck 1967] A. Grothendieck, "Éléments de géométrie algébrique, IV: Étude locale des schémas et des morphismes de schémas, IV", Inst. Hautes Études Sci. Publ. Math. 32 (1967), 5361. MR 39 \#220 Zbl 0153.22301

[Ishida 1978] M.-N. Ishida, "Compactifications of a family of generalized Jacobian varieties”, pp. 503-524 in Proceedings of the International Symposium on Algebraic Geometry (Kyoto, 1977), edited by M. Nagata, Kinokuniya Book Store, Tokyo, 1978. MR 81h:14025 Zbl 0415.14015

[Jarvis 2000] T. J. Jarvis, "Compactification of the universal Picard over the moduli of stable curves", Math. Z. 235:1 (2000), 123-149. MR 2001m:14040 Zbl 0980.14020

[Kleiman 2005] S. L. Kleiman, “The Picard scheme”, pp. 235-321 in Fundamental algebraic geometry: Grothendieck's FGA explained (Trieste, 2003), Math. Surveys Monogr. 123, Amer. Math. Soc., Providence, RI, 2005. MR 2223410 Zbl 1085.14001

[Kodaira 1960] K. Kodaira, "On compact analytic surfaces”, pp. 121-135 in Analytic functions (Princeton, NJ, 1958), Princeton Mathematical Series 24, Princeton University Press, 1960. MR 25 \#3939 Zbl 0137.17401

[Langer 2004a] A. Langer, "Moduli spaces of sheaves in mixed characteristic", Duke Math. J. 124:3 (2004), 571-586. MR 2005g:14082 Zbl 1086.14036

[Langer 2004b] A. Langer, "Semistable sheaves in positive characteristic", Ann. of Math. (2) 159:1 (2004), 251-276. MR 2005c:14021 Zbl 1080.14014

[Liu et al. 2004] Q. Liu, D. Lorenzini, and M. Raynaud, "Néron models, Lie algebras, and reduction of curves of genus one", Invent. Math. 157:3 (2004), 455-518. MR 2005m:14039 Zbl 1060.14037

[López-Martín 2005] A. C. López-Martín, "Simpson Jacobians of reducible curves", J. Reine Angew. Math. 582 (2005), 1-39. MR 2006d:14032 Zbl 1078.14033

[Maruyama 1996] M. Maruyama, "Construction of moduli spaces of stable sheaves via Simpson's idea”, pp. 147-187 in Moduli of vector bundles (Kyoto, 1994), edited by M. Maruyama, Lecture Notes in Pure and Appl. Math. 179, Dekker, New York, 1996. MR 97h:14020 Zbl 0885.14005 
[Mayer and Mumford 1964] A. Mayer and D. Mumford, "Further comments on boundary points", Lecture notes, Summer Institute on Algebraic Geometry (Woods Hole, MA), Amer. Math. Soc. Summer Institute, 1964, Available at http://www.jmilne.org/math/Documents/index.html.

[Mazur 1972] B. Mazur, "Rational points of abelian varieties with values in towers of number fields”, Invent. Math. 18 (1972), 183-266. MR 56 \#3020 Zbl 0245.14015

[Mazur 1977] B. Mazur, "Modular curves and the Eisenstein ideal", Inst. Hautes Études Sci. Publ. Math. 47 (1977), 33-186. MR 80c:14015 Zbl 0394.14008

[Mazur and Wiles 1984] B. Mazur and A. Wiles, "Class fields of abelian extensions of $\mathbb{Q}$ ", Invent. Math. 76:2 (1984), 179-330. MR 85m:11069 Zbl 0545.12005

[Melo and Viviani 2012] M. Melo and F. Viviani, "Fine compactified Jacobians", Math. Nachr. 285:8-9 (2012), 997-1031. Zbl 06049557

[Néron 1964] A. Néron, "Modèles minimaux des variétés abéliennes sur les corps locaux et globaux”, Inst. Hautes Études Sci. Publ.Math. 21 (1964), 5-128. MR 31 \#3423 Zbl 0132.41403

[Oda and Seshadri 1979] T. Oda and C. S. Seshadri, "Compactifications of the generalized Jacobian variety”, Trans. Amer. Math. Soc. 253 (1979), 1-90. MR 82e:14054 Zbl 0418.14019

[Pandharipande 1996] R. Pandharipande, "A compactification over $\bar{M}_{g}$ of the universal moduli space of slope-semistable vector bundles”, J. Amer. Math. Soc. 9:2 (1996), 425-471. MR 96f:14014 Zbl 0886.14002

[Raynaud 1970] M. Raynaud, "Spécialisation du foncteur de Picard”, Inst. Hautes Études Sci. Publ. Math. 38 (1970), 27-76. MR 44 \#227 Zbl 0207.51602

[Silverman 1994] J. H. Silverman, Advanced topics in the arithmetic of elliptic curves, Graduate Texts in Mathematics 151, Springer, New York, 1994. MR 96b:11074 Zbl 0911.14015

[Simpson 1994] C. T. Simpson, "Moduli of representations of the fundamental group of a smooth projective variety. I", Inst. Hautes Études Sci. Publ. Math. 79 (1994), 47-129. MR 96e:14012 Zbl 0891.14005

Communicated by Brian Conrad

Received 2011-09-23 Revised 2012-01-13 Accepted 2012-02-20

jkass@umich.edu

Institut für Algebraische Geometrie, Leibniz Universität Hannover, Welfengarten 1, D-30167 Hannover, Germany http://www2.iag.uni-hannover.de/ kass/ 


\section{Algebra \& Number Theory}

msp.org/ant

\section{EDITORS}

MANAGING EDITOR

Bjorn Poonen

Massachusetts Institute of Technology

Cambridge, USA

\author{
EDITORIAL BOARD CHAIR \\ David Eisenbud \\ University of California \\ Berkeley, USA
}

\section{BOARD OF EDITORS}

Georgia Benkart

Dave Benson

Richard E. Borcherds

John H. Coates

J-L. Colliot-Thélène

Brian D. Conrad

Hélène Esnault

Hubert Flenner

Edward Frenkel

Andrew Granville

Joseph Gubeladze

Ehud Hrushovski

Craig Huneke

Mikhail Kapranov

Yujiro Kawamata

János Kollár

Yuri Manin

Barry Mazur

Philippe Michel
University of Wisconsin, Madison, USA

University of Aberdeen, Scotland

University of California, Berkeley, USA

University of Cambridge, UK

CNRS, Université Paris-Sud, France

University of Michigan, USA

Freie Universität Berlin, Germany

Ruhr-Universität, Germany

University of California, Berkeley, USA

Université de Montréal, Canada

San Francisco State University, USA

Hebrew University, Israel

University of Virginia, USA

Yale University, USA

University of Tokyo, Japan

Princeton University, USA

Northwestern University, USA

Harvard University, USA

École Polytechnique Fédérale de Lausanne
Susan Montgomery

Shigefumi Mori

Raman Parimala

Jonathan Pila

Victor Reiner

Karl Rubin

Peter Sarnak

Joseph H. Silverman

Michael Singer

Vasudevan Srinivas

J. Toby Stafford

Bernd Sturmfels

Richard Taylor

Ravi Vakil

Michel van den Bergh

Marie-France Vignéras

Kei-Ichi Watanabe

Efim Zelmanov
University of Southern California, USA

RIMS, Kyoto University, Japan

Emory University, USA

University of Oxford, UK

University of Minnesota, USA

University of California, Irvine, USA

Princeton University, USA

Brown University, USA

North Carolina State University, USA

Tata Inst. of Fund. Research, India

University of Michigan, USA

University of California, Berkeley, USA

Harvard University, USA

Stanford University, USA

Hasselt University, Belgium

Université Paris VII, France

Nihon University, Japan

University of California, San Diego, USA

\section{PRODUCTION}

production@msp.org

Silvio Levy, Scientific Editor

See inside back cover or msp.org/ant for submission instructions.

The subscription price for 2013 is US \$200/year for the electronic version, and \$350/year ( $\$ 40$, if shipping outside the US) for print and electronic. Subscriptions, requests for back issues and changes of subscribers address should be sent to MSP.

Algebra \& Number Theory (ISSN 1944-7833 electronic, 1937-0652 printed) at Mathematical Sciences Publishers, 798 Evans Hall \#3840, c/o University of California, Berkeley, CA 94720-3840 is published continuously online. Periodical rate postage paid at Berkeley, CA 94704, and additional mailing offices.

ANT peer review and production are managed by EditFLOW ${ }^{\circledR}$ from Mathematical Sciences Publishers.

\section{PUBLISHED BY}

mathematical sciences publishers

nonprofit scientific publishing

http://msp.org/

(C) 2013 Mathematical Sciences Publishers 


\section{Algebra \& Number Theory}

$\begin{array}{lll}\text { Volume } 7 & \text { No. } 2 & 2013\end{array}$

The system of representations of the Weil-Deligne group associated to an abelian variety 243 RUTGER NOOT

Fourier-Jacobi coefficients of Eisenstein series on unitary groups

BEI ZHANG

The phase limit set of a variety

MoUnIR NisSE and FRANK SoTTILE

Base change behavior of the relative canonical sheaf related to higher dimensional moduli

Zsolt PATAKFALVI

Two ways to degenerate the Jacobian are the same

JESSE LEO KASS

Arithmetic motivic Poincaré series of toric varieties

Helena Cobo Pablos and Pedro Daniel González Pérez

Maximal ideals and representations of twisted forms of algebras

Michael LAU and ARTURo PiAnZOLA

Higher Chow groups of varieties with group action 